\title{
Discussion on Out-plane Stresses of the Simplified Elastic-plastic Constitutions for Plane Strain Problems
}

\author{
Changlu Tian ${ }^{1,}$, Ting $\mathrm{Wu}^{1, \mathrm{~b}}$ \\ ${ }^{1}$ Department of Mechanical Engineering, Jiangnan University, Wuxi, 214122, China \\ acltian63@163.com, b997784727@qq.com
}

Keywords: Hydrostatic stress, Effect of elasticity, Elastic-plastic fields

\begin{abstract}
The results demonstrate that the effect of elasticity in plastic zone on elastic-plastic fields is remarkable in many cases, while elastic volume deformation in plastic zone generally has been omitted in the solution procedures of the classical plastic theory due to the mathematic problems. The analytical results are further validated by finite element analyses, and it is concluded that the analogy results are physically more reasonable, especially in the existence of considerable hydrostatic stress.
\end{abstract}

\section{Introduction}

Most elastic-plastic solutions are based on the deformation theory of plasticity or the non-linear elasticity theory. However, to obtain the analytical solutions even for elastic-plastic plane strain problems is generally very difficulty. One problem is that the elastic-plastic constitutive law usually depends on out-of-plane stress $\sigma_{33}$ in a complicated manner and its elimination is not easy as reported [1]. Elastic deformation in elastic-plastic problems was taken into account have been reported [2], but the solution procedures and the expressions are general too complicated. For most relatively complex problems, such as elastic-plastic crack tip fields, the incompressible or full plastic assumption has been are employed to simplify the constitutive law. So it is assumed that $\sigma_{33} /\left(\sigma_{11}+\sigma_{22}\right)=0.5$ in plastic zone [3-7], and $\sigma_{33} /\left(\sigma_{11}+\sigma_{22}\right)=v$ in elastic zone (where $\sigma_{33}$ is out-of-plane stress, $\sigma_{11}, \sigma_{22}$ are in-plane stresses, and $v$ is Poisson's ratio).

However, the incompressible or full plastic results are the limited cases of real elastic-plastic fields, and they are applicable only on the condition that mean stresses are comparatively very low. The effect of elasticity in elastic-plastic crack-tip fields was reported [8-11], but in general, the error due to the incompressible or full plastic assumption is unclear. The current study focuses on investigating the effect of elasticity in plastic zone on elastic-plastic fields.

\section{Simplification of Plane Strain Elastic-Plastic Problems}

Elastic-plastic plane strain problems for the deformation theory of plasticity are considered. For simplification, the bi-linear elastic-plastic model is studied. The stress-strain relation in multiaxial stress state is in the form of

$$
\bar{\varepsilon}_{i j}=(1+v) \bar{S}_{i j}+(1-2 v) \bar{\sigma}_{k k} \delta_{i j}+\frac{3}{2}\left(\frac{E}{E_{p}}-1\right)\left(1-\bar{\sigma}_{e}^{-1}\right) \bar{S}_{i j},
$$

where, $\sigma_{o}$ is the initial yield stress and $\varepsilon_{o}=\sigma_{o} / E$ is the corresponding strain, $E_{p}$ denotes the tangent modulus of elastic-plastic stress-strain curve (i.e., the slope of the line as $\sigma>\sigma_{o}$ ), $v$ is Poisson's ratio, the strain components $\varepsilon_{i j}$ are related to the deviation stress components $S_{i j}$ of stress tensor $\sigma_{i j}, S_{i j}=\sigma_{i j}-\sigma_{m} \delta_{i j}$, and $\bar{\sigma}_{e}=\sigma_{e} / \sigma_{o}, \bar{S}_{i j}=S_{i j} / \sigma_{o}$ all parameters with overbars are dimensionless ones throughout this paper.

Some different cases of elastic-plastic fields referred in literature are discussed firstly. As 
$\bar{\sigma}_{e}=\sigma_{e} / \sigma_{o} \rightarrow \infty$, Eq. (1) is simplified as

$$
\varepsilon_{i j}=\frac{1+v}{E} S_{i j}+\frac{1-2 v}{3 E} \sigma_{k k} \delta_{i j}+\frac{3}{2}\left(\frac{1}{E_{p}}-\frac{1}{E}\right) S_{i j} .
$$

In this case, the out-plane stress is

$$
\sigma_{33}=\frac{E_{P}}{E}\left[v+0.5 \cdot\left(n_{p}-1\right)\right]\left(\sigma_{11}+\sigma_{22}\right) \text {. }
$$

The crack tip solution by employing Eq. (2) was reported [9]. Moreover, if the first two elastic terms are omitted (i.e. $\varepsilon_{i j}^{e}=0 ; \varepsilon_{i j}=\varepsilon_{i j}^{p}$ ), the full plastic constitution is obtained,

$$
\begin{aligned}
& \varepsilon_{i j}=\frac{3}{2}\left(\frac{1}{E_{p}}-\frac{1}{E}\right) S_{i j} . \\
& \sigma_{33}=0.5 \cdot\left(\sigma_{11}+\sigma_{22}\right) .
\end{aligned}
$$

The bi-linear crack tip solution by employing incompressible model of Eq. (4) was reported by Hutionson [3]. The full plastic model assumes that elastic strain is zero and plastic strain is the total strain, and also that materials are incompressible.

The cases for bi-linear materials are discussed above. Similarly, the crack tip solutions for power-law materials by employing incompressible model were also reported by many literatures [3-7].

\section{The Out-plane Stresses of Plane Strain Elastic-Plastic Problems}

For plane strain elastic-plastic problems, the constitutive law usually depends on $\sigma_{3}$ in a complicated manner and its elimination is not equally easy [1]. Generally, in elastic-plastic condition, out-of-plane stresses should be

$$
\sigma_{33}=v_{e p}\left(\sigma_{11}+\sigma_{22}\right)
$$

where, $v_{e p}$ is a parameter for elastic-plastic plane strain problems. As $\bar{\sigma}_{e}=\sigma_{e} / \sigma_{o} \leq 1.0$ ( i.e., in linear elastic condition), there is $v_{e p}=v$. The effective stress $\sigma_{e}$ is in the form of

$$
\sigma_{e}=(1 / \sqrt{2}) \cdot\left[\left(\sigma_{11}-\sigma_{22}\right)^{2}+\left(\sigma_{22}-\sigma_{33}\right)^{2}+\left(\sigma_{33}-\sigma_{11}\right)^{2}\right]^{\frac{1}{2}} \text {. }
$$

By substituting Eq. (6) into (7), a relation between $\sigma_{e}$ and $v_{e p}$ is obtained,

$$
\bar{\sigma}_{e}=\sqrt{k^{2}-k+1-(k+1)^{2}\left(v_{e p}-v_{e p}^{2}\right)} \cdot \bar{\sigma}_{11} .
$$

Here $k=\sigma_{22} / \sigma_{11}$ (by assuming $\sigma_{11} \geq \sigma_{22}$ ), and its different values represent different stress states of the biaxial elements. For plane strain problems, with $\varepsilon_{33}=0$, the following relation could be directly derived from Eq. (1).

$$
v_{e p}=\frac{\sigma_{3}}{\sigma_{1}+\sigma_{2}}=\frac{v+\left(E / E_{P}-1\right)\left(1-\bar{\sigma}_{i}^{-1}\right) / 2}{1+\left(E / E_{P}-1\right)\left(1-\bar{\sigma}_{i}^{-1}\right)},
$$

So a general relation between $\sigma_{e}$ and $v_{e p}$ were obtained [11], it can be rewritten as

$$
\bar{\sigma}_{e}=\bar{\sigma}_{e}\left(n_{p}, v_{e p}\right)=\frac{\left(E / E_{p}-1\right)\left(0.5-v_{e p}\right)}{\left(E / E_{p}-1\right)\left(0.5-v_{e p}\right)+v-v_{e p}} .
$$


By combining of these two $v_{e p} \sim \sigma_{e}$ relations leads to

$\left(\frac{q}{\sigma_{o}}\right) \sqrt{k^{2}-k+1-(k+1)^{2}\left(v_{e p}-v_{e p}^{2}\right)}=\frac{\left(E / E_{p}-1\right)\left(0.5-v_{e p}\right)}{\left(E / E_{p}-1\right)\left(0.5-v_{e p}\right)+v-v_{e p}} \geq 1.0$,

it can be expressed as

$v_{e p}=v_{e p}\left(\sigma_{11}, k, E / E_{p}, \sigma_{0}, v\right) \quad$ or $\quad v_{e p}=v_{e p}\left(\sigma_{11}, \sigma_{22}, E / E_{p}, \sigma_{0}, v\right)$.

The value of $v_{e p}$ is determined by Eq. (10a) for different values $k$ separately. The materials constants are $v=0.3, E=200 \mathrm{GPa}, n_{p}=E / E_{P}=5,20$ and $\varepsilon_{o}=\sigma_{o} / E=0.002$. The curves of $v_{e p}$ versus $k$ of different stress states are shown in Fig. 1. It is shown that the values of $v_{e p}$ is dependent on stress states. All the $v_{e p}$ values for two kinds of materials cannot reach the incompressible values of 0.5 , especially as $k=\sigma_{22} / \sigma_{11} \rightarrow 1.0$.

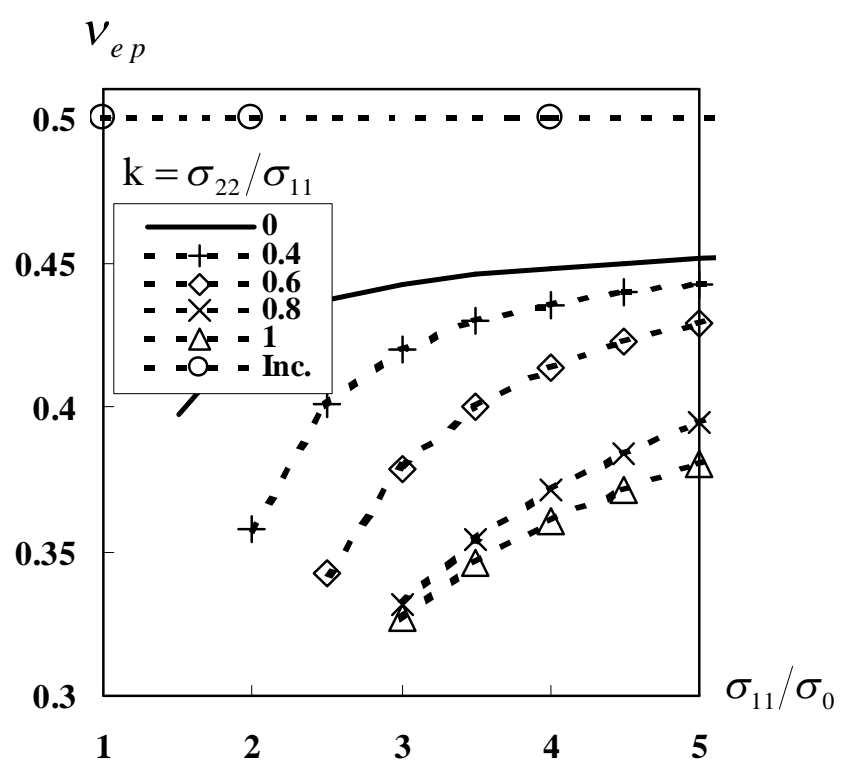

(a) $E / E_{P}=5$

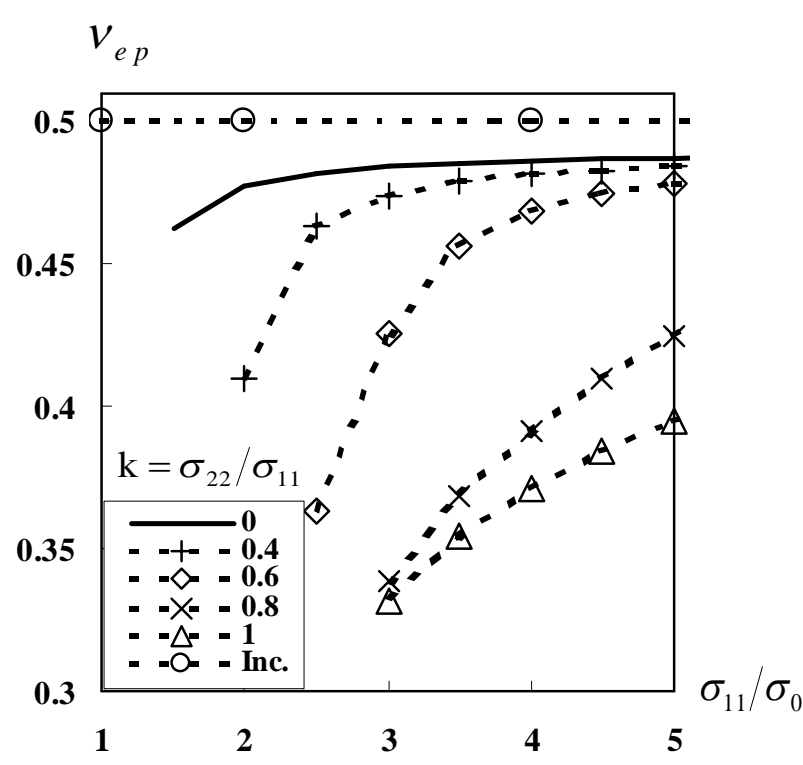

(b) $E / E_{P}=20$

Fig. 1 Elastic-plastic Poisson's ratio $v_{e p}$ of plane strain elements for different stress states.

By Eq. (6) and Eq. (5), we could obtain $\sigma_{33}^{\text {Inc. }} / \sigma_{33}=0.5 / v_{e p}$. So the deflections of $\sigma_{33}$ due to the incompressible assumption can be evaluated by the ratio of $0.5 / v_{e p}$. With the $v_{e p}$ values in Fig. 1 , it can be found that the deflections are considerable, especially as $k=\sigma_{22} / \sigma_{11} \rightarrow 1$. The values of out-of-plane stress could be overestimated above $50 \%$ at most for two different materials $\left(E / E_{P}=5,20\right)$.

By substituting the equation of $\sigma_{33}=0.5 \cdot\left(\sigma_{11}+\sigma_{22}\right)$ into effective stress Eq. (7). It leads to the well-known incompressible equation of

$$
\sigma_{e}=\left[(3 / 4)\left(\sigma_{11}-\sigma_{22}\right)^{2}\right]^{\frac{1}{2}} \text {. }
$$

The deflections of effective stresses $\sigma_{e}$ due to the incompressible assumption are also remarkable. Substituting Eq. (10) into Eq. (8), we get the distributions of effective stresses. For a typical case, $k=\sigma_{22} / \sigma_{11}=1.0, \bar{\sigma}_{12}=0, E / E_{P}=5,20$, the values of $\sigma_{e}$ are plotted in Fig. 2. 


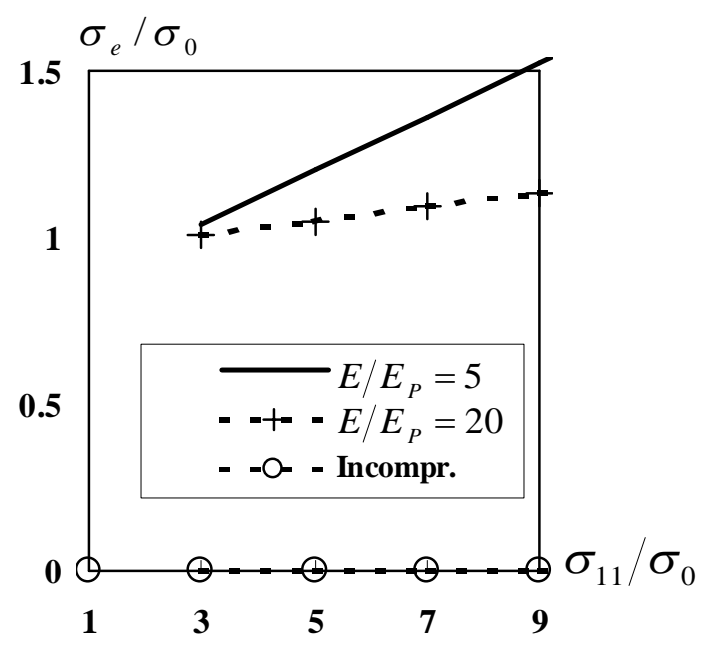

Fig. 2 The effective stresses of elastic-plastic plane strain elements in biaxial tension.

It is seen that the values of $\sigma_{e}$ of Eq. (11) keeps zero, while the values of $\sigma_{e}$ of the analogy results is dependent on elastic, plastic material data $E, E_{P}$ as well as load levels of $\sigma_{11} / \sigma_{0}$. This phenomenon means that so-called "incompressible materials" would never yield for the case of $k=\sigma_{22} / \sigma_{11}=1.0$ under plane strain condition, and neither plastic nor elastic deformation would develop.

\section{Concluding Remark}

The present analysis is limited to the deformation plasticity theory (or the non-linear elasticity). This work investigates the basic issues in the classical plasticity because it is very difficult to get an analytical solution if the volume deformation related to hydrostatic stress were not omitted in its solution procedure.

Some results of elastic-plastic plane strain problems are presented. It is concluded that the elastic volume deformation developed in the so-called plastic zone is important to failure mechanism in many cases, and further theoretical study is needed so as to develop the scheme for establishing more convincing methodology to obtain the real elastic-plastic fields.

\section{References}

[1] A. Capsoni, L. Corradi. Variational formulations for the plane strain elastic-plastic problem for materials governed by the von Mises criterion. Int. J. Plast. 12(4) (1996) 547-560.

[2] A. N. Eraslan. Von Misses' yield criterion and nonlinearly hardening rotating shafts. Acta. Mech. 168(3) (2004) 129-144.

[3] J. W. Hutchinson. Singular behavior at the end of a tensile crack in a hardening material. J. Mech. Phys. Solids. 16 (1968) 13-31.

[4] J. R. Rice, G. R. Rosengren. Plane strain deformation near a crack tip in a power -law hardening material. J. Mech. Phys. Solids. 16 (1968) 1-12.

[5] Y. C. Li, Z. Q. Wang. High-order asymptotic field of tensile plane-strain nonlinear crack problems. Sci. Sinica A 29 (1986) 941-955.

[6] F. Ma, M. A. Sutton, X. Deng. Plane strain mixed mode crack-tip stress fields characterized by a triaxial stress parameter and a plastic deformation extent based characteristic length. J. Mech. Phys. Solids. 49(12) (2001) 2921-2953. 
[7] X. K. Zhu, Y. J. Chao. Constraint effects on crack-tip fields in elastic-plastic perfectly plastic materials. J. Mech. Phys. Solids. 49(2) (2001) 363-399.

[8] M. S. Sharma, N. L. Aravas. Determination of higher-order terms in asymptotic elastoplastic crack tip solution. J. Mech. Phys. Solids. 39(8) (1991) 1043-1072.

[9] S. Yang, Y. J. Chao. Asymptotic deformation and stress fields at the tip of a sharp notch in an elastic-plastic material. Int. J. Fract. 54(3) (1992) 211-224.

[10]C. L. Tian, J. Wen. Non-uniform distribution of energy density near a crack tip in the framework of HRR model. Int. J. Fract. 110(4) (2001) 45-50.

[11]C. L. Tian, Y. X. Gao. Role of elasticity in elastic-plastic fracture. Int. J. Solid. Struct. 42(3) (2005) 951-970. 